\title{
The Raman coupling function in amorphous silica and the nature of the long wavelength excitations in disordered systems.
}

\author{
A. Fontana ${ }^{1}$, R. Dell'Anna ${ }^{1}$, M. Montagna ${ }^{1}$, F. Rossi $^{1}$, G. Viliani ${ }^{1}$, \\ G. Ruocco ${ }^{2}$, M. Sampoli ${ }^{3}$, U. Buchenau ${ }^{4}$, and A. Wischnewski ${ }^{4}$. \\ 1 Universitá di Trento and Istituto Nazionale di Fisica della Materia, I-38050, Povo, Trento, \\ Italy. \\ 2 Universitá de L'Aquila and Istituto Nazionale di Fisica della Materia, I-67100, L'Aquila, Italy. \\ ${ }^{3}$ Universitá di Firenze and Istituto Nazionale di Fisica della Materia, I-50139, Firenze, Italy. \\ ${ }^{4}$ Institut fur Festkörperforschung, Forschungszentrum, Jülich, Postfach 1913, D-52425, Jülich, \\ Germany.
}

\begin{abstract}
New Raman and incoherent neutron scattering data at various temperatures and molecular dynamic simulations in amorphous silica, are compared to obtain the Raman coupling coefficient $C(\omega)$ and, in particular, its low frequency limit. This study indicates that in the $\omega \rightarrow 0$ limit $C(\omega)$ extrapolates to a non vanishing value, giving important indications on the characteristics of the vibrational modes in disordered materials; in particular our results indicate that even in the limit of very long wavelength the local disorder implies non-regular local atomic displacements.
\end{abstract}


The vibrational dynamics in topologically disordered systems is one of the most intriguing problems of present-day condensed matter physics [1]. In this respect, an important issue is the excess of vibrational states, that has been shown to exist in glasses at low frequencies [2] by: (i) the specific heat that, above a few degrees Kelvin, exhibits a bump in $C_{P} / T^{3}$ [3] 5 and (ii) the total density of states $g(\omega)$ that, at corresponding frequencies, shows a broad excess band in the plot of $g(\omega) / \omega^{2}$ [6 B] often referred to as boson peak (BP). Both findings indicate a non-Debye behaviour of the vibrational properties at frequencies below $10 \div 50 \mathrm{~cm}^{-1}$, depending on the specific glass. The nature of the excess modes is still object of speculations [1]. In particular, in the case of $\mathrm{SiO}_{2}$ there are two prevailing hypotheses. According to the first one, strong phonon scattering by the structural disorder induces a localization of the vibrational states, and the excess of states appears at the crossover between the low frequency propagating and the high frequency localized modes [9]. In the other picture, collective propagating modes persist up to frequencies higher than the BP frequencies, and the BP itself reflects their density of states [10 12].

Spectral information on the BP is usually obtained by Inelastic Neutron Scattering (INS) or by Raman scattering (RS) spectra. In both cases, the first order scattering intensity, $I_{(R, N)}(\omega, T)$, is connected to the vibrational density of states by [13]:

$$
I_{(R, N)}(\omega, T) \propto \frac{n(\omega)+1}{\omega} g(\omega) C_{(R, N)}(\omega)
$$

here $n(\omega)$ is the Bose population factor and $C_{(R, N)}(\omega)$ is the probe-excitations coupling function. The incoherent neutron scattering depends only on the absolute motion of atoms in space, so $C_{N}(\omega)=1$. On the contrary, RS detects the relative displacement of neighbouring atoms and $C_{R}(\omega)$ turns out to be a complicated function of $\omega$ [13]. Since $C_{R}(\omega)$ is usually unknown, it results from Eq. 1 that RS spectra are not sufficient to determine $g(\omega)$, though this experimental technique is excellent for luminosity and resolving power.

On a theoretical ground, different models have been developed for the frequency dependence of $C(\omega)$. In particular, it was shown that $i$ ) for slightly distorted plane wave vibrations, $C(\omega) \propto \omega^{2}$ [14]; ii) within the framework of the soft potential model [15], $C(\omega)=$ const; and 
iii) the fracton-like model implies $C(\omega) \propto \omega^{\alpha}$ [16, 17]. It is worth to note that all the models proposed so far, predict a power-law behaviour for $C(\omega)$. On the contrary, the results of numerical simulations are much more complex and rarely produce scaling behaviour, even on simple model systems 18 21.

On the experimental side, the direct comparison between RS and INS spectra appears to be a reliable procedure to determine $C(\omega)$, and then to check the validity of the proposed theoretical models. Though some attempts in this direction were made in the past [22], a definite conclusion as to the shape of $C(\omega)$ has not been reached [8, 17,23], mainly due to the presence of "spurious" effects in RS (namely, the presence of quasi-elastic scattering (QES) or of luminescence).

The aim of the present work is to determine the spectral shape of $C(\omega)$ in vitreous silica in the acoustic frequency range $\left(\omega<150 \mathrm{~cm}^{-1}\right)$, at different temperatures from new RS and INS measurements. The luminescence is carefully analysed and eliminated from RS spectra. The $I_{R} / I_{N}$ ratio results to be T-independent, except in the low frequency region where the effects of QES in RS are also evident. The coupling function, as defined in Eq.] for harmonic excitations, is then extrapolated as the $T \rightarrow 0$ limit of $I_{R} / I_{N}$. The obtained function does not show the power-law behaviour previously proposed in the theoretical models. Further, it appears not to vanish in the $\omega \rightarrow 0$ limit, giving important information on the characteristics of the normal modes of vibration in disordered systems. These findings are also compared with the outcoming of a molecular dynamics study of vitreous silica, which confirms the experimental result and allows a direct inspection of the vibrational modes.

The $\mathrm{SiO}_{2}$ suprasil sample purchased from Goodfellow was used for light scattering, while Heralux quartz from Heraeus, was used for the neutron scattering experiments. The Raman scattering measurements were performed using a standard Raman laser system. Depolarized spectra in $90^{\circ}$ scattering geometry were collected in the $-300 \div 5000 \mathrm{~cm}^{-1}$ frequency range. Such a wide range was necessary to take properly into account the shape and the temperature dependence of the luminescence background which is weak at room temperature, but becomes important for a quantitative determination of the coupling function at low fre- 
quency $\left(<20 \mathrm{~cm}^{-1}\right)$ and low temperature $(<70 \mathrm{~K})$. In our case the maximum luminescence contribution to the intensity in the problematic range $<7 \mathrm{~cm}^{-1}$ was estimated to be $\approx 7 \%$ at $12 \mathrm{~K}$ and $\approx 1 \%$ at $50 \mathrm{~K}$. The details of the data analysis and of the luminescence subtraction will be described in a more extended paper [24]. The neutron scattering data were taken at two different spectrometers (the time of flight spectrometer IN6 of the ILL at Grenoble, and a triple axis spectrometer at the Oak Ridge High Flux Reactor). The data underwent the usual corrections (subtraction of the empty container, normalization of different detectors to a vanadium measurement), and the density of vibrational states was derived following the procedure described in detail in Ref. [25]. Standard microcanonical molecular dynamics (MD) simulations of vitreous silica were performed on systems of $N=1536$ and $N=5148$ atoms (box lengths $L \approx 2.9$ and $4.3 \mathrm{~nm}$ respectively) interacting via the two- and three-body potential proposed by Vashista et al. [26]. The long range interaction was treated by the tapered reaction field method [27] and the equations of motion were integrated by the leap-frog [28] alghoritm with a time step $\Delta t=0.5 \mathrm{fs}$ (more details on the MD can be found in [29]). We used the normal mode analysis in the harmonic approximation and MD to derive the dynamical quantities of interest, i.e. the density of vibrational states $g(\omega)$ and the Raman spectrum $I_{R}(\omega)$. The computation of the Raman scattering needs a model for the effective atomic polarizability and its dependence on the relative atomic position. In this study we associated a point polarizability to each atom $\left(\alpha_{O} / \alpha_{S i}=4\right)$ and used the electric dipole propagator $\left(T^{(2)}(\vec{r})\right)$ to describe the effective atomic polarizability, $\Pi_{i}=\sum_{j} \alpha_{i} \alpha_{j} T^{(2)}\left(\vec{r}_{i}-\vec{r}_{j}\right)$. Even though this polarization model may be a crude approximation it gives a reasonable agreement with the experimental data. Further details of the calculation will be given in [24].

In Fig. 1 we report the Raman and neutron reduced spectra $J_{(R, N)}(\omega, T)$ :

$$
J_{(R, N)}(\omega, T)=\frac{I_{(R, N)}(\omega, T)}{\omega[n(\omega)+1]},
$$

that, according to Eq. (1), correspond to $g(\omega) C(\omega) / \omega^{2}$. The data reported in Fig. 1 show that the frequency dependence of the spectra obtained by the two techniques is similar at 
all the investigated temperatures. Indeed, at room temperature, the maximum of the boson peak $\left(\omega_{B P}\right)$ is centered at $\omega_{B P} \approx 45 \mathrm{~cm}^{-1}$ and $35 \mathrm{~cm}^{-1}$ in the RS and INS spectra respectively and the small blue shift of the RS maximum is due to the coupling function. Moreover, the temperature dependence of $\omega_{B P}$ was recently investigated in connection with the results of inelastic x-ray scattering study [12]. By increasing $T$, the BP frequencies, as seen by RS and INS, increase at the same rate, and at the same rate as the collective excitations at $Q=1.6 \mathrm{~nm}^{-1}$ observed by inelastic X-ray scattering. This temperature dependence was temptatively ascribed to a next-nearest neighbour fourth-order anharmonicity [12].

The ratios of Raman to neutron data collected at different temperatures in the range $50 \div 1100 \mathrm{~K}$ are reported in Fig. 2 where it is evident that the data at all temperatures are practically coincident for $\omega>\approx 30 \mathrm{~cm}^{-1}$, while the presence of non negligible QES produces a marked $T$ dependence in the low frequency region. The QES has been from time to time assigned to relaxation process, anharmonicity or multi-phonons scattering; its origin is beyond the purpose of the present work. As a consequence of QES, it is only in the $T \rightarrow 0$ limit that the $I_{R} / I_{N}$ ratio can be identified, apart from a proportionality factor, with the coupling function $C(\omega)$. However, by reducing the temperature, also in this low frequency region the data appear to pile up on the continuation of the straight line that fits the frequency behaviour in the $30-80 \mathrm{~cm}^{-1}$ range. The inset in Fig. 1 shows that above $\approx 20 \mathrm{~cm}^{-1}$ the 50 and $12 \mathrm{~K}$ spectra coincide, indicating that QES is negligible at these frequencies.

In Fig. 3, we compare the MD simulated coupling function with the experimental one at the lowest temperature. The MD data have the advantage of being free from QES contributions, but below $30 \mathrm{~cm}^{-1}$, owing to the finite size of the simulation box, the statistics is too poor to give reliable results [30]. In the reported range the agreement between simulated and experimental results is very satisfactory. As mentioned, the experimental data at $T=51$ $\mathrm{K}$ were fitted by a straight line in the $30-80 \mathrm{~cm}^{-1}$ range (full line in Fig. 3 ). We note that our coupling function does not follow a simple power-law, and seems to extrapolate to a non-vanishing value at $\omega \rightarrow 0$ as emphasized by the dashed lines in Fig. 3 that delimit the 
$\pm 2 \sigma$ confidence band of the fit.

The non vanishing value of $C(\omega \rightarrow 0)$ gives information on the characteristics of the vibrational modes in topologically disordered systems. It is well know that in crystalline materials the eigenvectors of the modes are plane waves, i. e. $\vec{e}_{j}(p) \propto \exp \left(i \vec{k}_{p} \cdot \vec{r}_{j}\right)$ where $j$ labels the atoms and $p$ the different modes which have well defined wavenumbers $k_{p}$. In the acoustic region the coupling function behaves as $C(\omega) \sim \omega^{2}[14]$. This $\omega^{2}$ law can be understood by considering that the scattering intensity is proportional to the square of the relative displacements of atoms, the main contribution coming from nearest neighbours; for the $p$-th mode this relative displacement is proportional to $\left|\vec{e}_{j}(p)-\vec{e}_{j-1}(p)\right| \approx\left|\nabla e_{j}(p)\right| \propto k_{p}$, and hence to $\omega_{p} / v$. At variance with the crystalline case, in a topologically disordered system the eigenvectors of the modes are no longer pure plane waves. As suggested by recent MD calculations on a model LJ glass [31,32] and on a model vitreous silica [33], the eigenvector of the $p$-th mode consists of a plane wave part, with a rather well defined wavenumber $k_{p}$, and of a further component $\epsilon_{j}$, whose characteristics are not correlated with $k_{p}$, and strongly resembling a white "noise" in the atomic displacements, $\vec{e}_{j}(p)=\vec{e} \sqrt{1-\sigma_{p}^{2}} \exp \left(i \vec{k}_{p} \cdot \vec{r}_{j}\right)+\sigma_{p} \vec{\epsilon}_{j}$. The plane-wave part accounts for the (rather) well defined peak observed in the dynamical structure factor of glasses, while the random-like part is the one mainly responsible for the value of $C(\omega)$. Indeed, at low frequencies, the local strain associated with the random-like component is much larger than that associated with the plane wave component. The same scenario is found in our MD simulation of $\mathrm{v}-\mathrm{SiO}_{2}$. In Fig. 4 we report as an example the $z$ component of the eigenvector of a normal mode $\left(\omega_{p} \approx 80 \mathrm{~cm}^{-1}\right)$ as a function of the $z$ atomic coordinate in the $N=1536$ atoms system. The plane wave component of this mode corresponds to a longitudinal mode, and superimposed to it there is the noisy part. Similar results concerning the characteristics of the normal modes in v-SiO 2 were recently obtained by Taraskin and Elliott (Fig. 4 of ref. [33]). Whether the weight of this noisy component $\left(\sigma_{p}\right)$ remains finite even at macroscopic wavelength, or, on the contrary, becomes negligible at $k_{p} \rightarrow 0$, is still matter of discussion. The MD, due to the finite size of the simulation box, cannot access the very low frequeny region, and therefore no information can be obtained on 
the magnitude of the noisy-to-plane wave ratio at long wavelengths. Indirect information on this issue can be gathered from the behaviour of $C(\omega)$ : in fact, a finite value for $C(\omega \rightarrow 0)$, as obtained in this work, supports the conjecture that the noisy component does not disappear even in the long wavelength limit. In fact, this can be the only origin of a non vanishing coupling coefficient at low frequency, because as mentioned above, the contribution of the plane wave component to $C(\omega)$, which is proportional to $\omega^{2}$, vanishes in the $\omega \rightarrow 0$ limit.

Such conjecture is also supported by recent Brillouin light scattering studies of the tails of the inelatic peaks in the dynamic structure factors $S(Q, \omega)$ of glasses. In Brillouin light scattering $\left(Q \approx 0.02-0.04 \mathrm{~nm}^{-1}\right)$ 34, 35 the $S(Q, \omega)$ shows a $\omega$-independent tail on the low frequency side of the inelastic peaks, whose value is $Q$-independent. This implies that, no matter how low the $Q$ value is, the eigenvectors of all the modes at frequency smaller than the peak frequency, $\Omega_{p}=v Q$, contain in their space Fourier transform such a $Q$ component. In other words, the eigenvector of a mode at frequency $\Omega$, contains in its space Fourier transform all the $Q$ components larger than $Q_{p}=\Omega / v$. This is verified down to the lowest experimentally investigated frequency $\left(\omega \approx 0.001 \mathrm{~cm}^{-1}[34]\right)$ corresponding to a wavelength of $\approx 10 \mu \mathrm{m}$.

This result seems to contradict a well established, and textbook- reported, idea: "At wavelengths $\lambda$, much higher than the interatomic distance $a$ and the local order correlation length $\xi$, the sound wave feels the disordered system as homogeneous and all happens as in a continuous elastic medium". The present results indicate that this picture needs to be revised at a microscopic level. Even when $\lambda / \xi$ is large, the modes are not pure plane waves: the local disorder still implies non-regular local atomic displacements.

We thank S. Elliott for a preprint of Ref. [33 and for useful discussions. 


\section{REFERENCES}

[1] For a review see, Philosophical Magazine B77, No. 2, (1998), special issue: Sixth International Workshop on Disordered Systems, Andalo, 1997, guest editors A. Fontana and G. Viliani.

[2] For a review see, Amorphous Solids : Low-Temperature properties, edited by W.A. Phillips, (Springer, Berlin, 1981).

[3] A. P. Sokolov, A. Kisliuk, D. Quitmann, and E. Duval, Phys. Rev B48, 7692 (1993).

[4] A. Brodin, A. Fontana, L. Borjesson, G. Carini and L.M. Torell, Phys. Rev. Lett. 73, 2067 (1994).

[5] G. Carini et al., Phys. Rev. B52, 9342 (1995).

[6] U. Buchenau et al., Phys. Rev. 34, 5665 (1986).

[7] U. Buchenau, H.M. Zhou, N. Nucker, K.S. Gilroy, and W.A. Phillips, Phys. Rev. Lett. 60, 1318 (1988).

[8] A.P. Sokolov, U. Buchenau, W. Steffen, B. Frick, and A. Wischmewsski, Phys. Rev. B52, R9815 (1995).

[9] M. Foret, E. Courtens, R. Vacher, J.B.Suck, Phys. Rev. Lett. 78, 4670 (1997).

[10] P. Benassi et al., Phys. Rev. Lett., 77, 3835 (1996).

[11] C. Masciovecchio et al., Phys. Rev. B55, 8049 (1997).

[12] C. Masciovecchio et al., preprint.

[13] F.L. Galenneer, and P.L. Sen, Phys. Rev. B17, 1928 (1978).

[14] A.J. Martin and W. Brenig, Phys. Status Solidi (B) 64, 163 (1972).

[15] V.L. Gurevich, D.A. Parshin, J. Pelous, and H.R. Schober, Phys. Rev. B48, 16318 (1993). 
[16] A. Fontana, F. Rocca, and M.P. Fontana, Phys. Rev. Lett. 58, 503 (1987).

[17] A. Fontana, F. Rocca, M.P. Fontana, B. Rosi, and A.J. Dianoux, Phys. Rev. B41, 3778 (1990).

[18] M. Montagna et al., Phys. Rev. Lett. 65, 1136 (1990).

[19] V. Mazzacurati et al., Phys. Rev. B45, 2126 (1992).

[20] G. Viliani et al., Phys. Rev. B52, 3346 (1995).

[21] O. Pilla, G. Viliani, R. Dell'Anna, and G. Ruocco, Physica A247, 23 (1997).

[22] A. Mermet, N.V. Surovtsev, E. Duval, J.F. Jal, and A.J. Dianoux, Europhys. Lett. 36, $277(1996)$.

[23] T. Achibat, A. Boukenter, and E. Duval, J. Chem. Phys. 99, 2046 (1993).

[24] M. Montagna et al., to be published.

[25] A. Wischnewski, U. Buchenau, A.J. Dianoux, W.A. Kamitakahara, and J.L. Zaretsky, Phil. Mag. B77, 579 (1998).

[26] P. Vashista, R.K. Kalia, J.P. RinoD, and I. Ebbsjö, Phys. Rev. B41, 12197 (1990).

[27] G. Ruocco and M. Sampoli, Molec. Phys. 82, 875 (1994).

[28] M.P. Allen and D.J. Tildesley, Computer Simulation of Liquids, Oxford University Press, (N.Y. 1990).

[29] R. Dell'Anna, G. Ruocco, M. Sampoli, and G. Viliani, Phys. Rev. Lett 80 , 1236 (1998).

[30] M. Montagna, P. Benassi, W. Frizzera, O. Pilla, G. Viliani, V. Mazzacurati, G. Ruocco, G. Signorelli, Physica A191, 348 (1992)

[31] V. Mazzacurati, G. Ruocco and M. Sampoli, Europhys. Lett. 34, 681 (1996).

[32] M. Sampoli, P. Benassi, R. Dell'Anna, V. Mazzacurati, and G. Ruocco, Phil. Mag. B77, 
473 (1998).

[33] S.N. Taraskin, and S.R. Elliot, preprint.

[34] G. Monaco, G. Ruocco, L. Comez, and D. Fioretto, J. Non Cryst. Sol. 00, 0000 (1998);

G. Monaco, D. Fioretto, C. Masciovecchio, G. Ruocco, and F. Sette, preprint.

[35] P. Benassi, A. Fontana, V. Mazzacurati, and M. Sampoli, preprint. 


\section{CAPTIONS}

Fig. 1 - (a): Reduced Raman spectra, $I(\omega) /\{\omega[n(\omega)+1]\}$, in the $0-150 \mathrm{~cm}-1$ range at different temperatures (from top to bottom 45, 323, 423, 523, 873, and $1073 \mathrm{~K}$ ). (b): Density of states divided by $\omega^{2}$ as obtained by inelastic neutron scattering in the frequency range as in a) at different temperatures (from top to bottom 51, 318, 523, 873, and $1104 \mathrm{~K}$ ). The dashed line is the calculated Debye contribution as obtained using the sound velocities determined by Brillouin scattering at $50 \mathrm{~K}$. (c): Reduced Raman spectra (in arbitrary units) at 12 and $50 \mathrm{~K}$

Fig. 2 - $C(\omega)$ obtained by the Raman to Neutron scattering ratio at some significant temperatures (from top to bottom $51(\circ), 224(\bullet), 271(\times), 318(\diamond), 423(\triangle), 523(\star), 873(\nabla)$, and $1104(+) \mathrm{K})$. The dashed line is the linear fit to the $51 \mathrm{~K}$ data in the $30-80 \mathrm{~cm}^{-1}$ range. The inset shows the low frequency part of $C(\omega)$.

Fig. 3 - Comparison between $C(\omega)$ obtained by simulation (full circles) and experiments at $T=51 \mathrm{~K}$ (open circles). The full line is the linear fit to the experimental data in the $30-80 \mathrm{~cm}^{-1}$ range, and the dashed lines delimit the $\pm 2 \sigma$ confidence band.

Fig. 4 - The z-components of the eigenvector of the mode at $\omega=80 \mathrm{~cm}^{-1}$, obtained by $\mathrm{MD}$ of $\mathrm{SiO}_{2}$, are reported versus the z-component of the atomic coordinates. The continuous line is the sine best fit. 


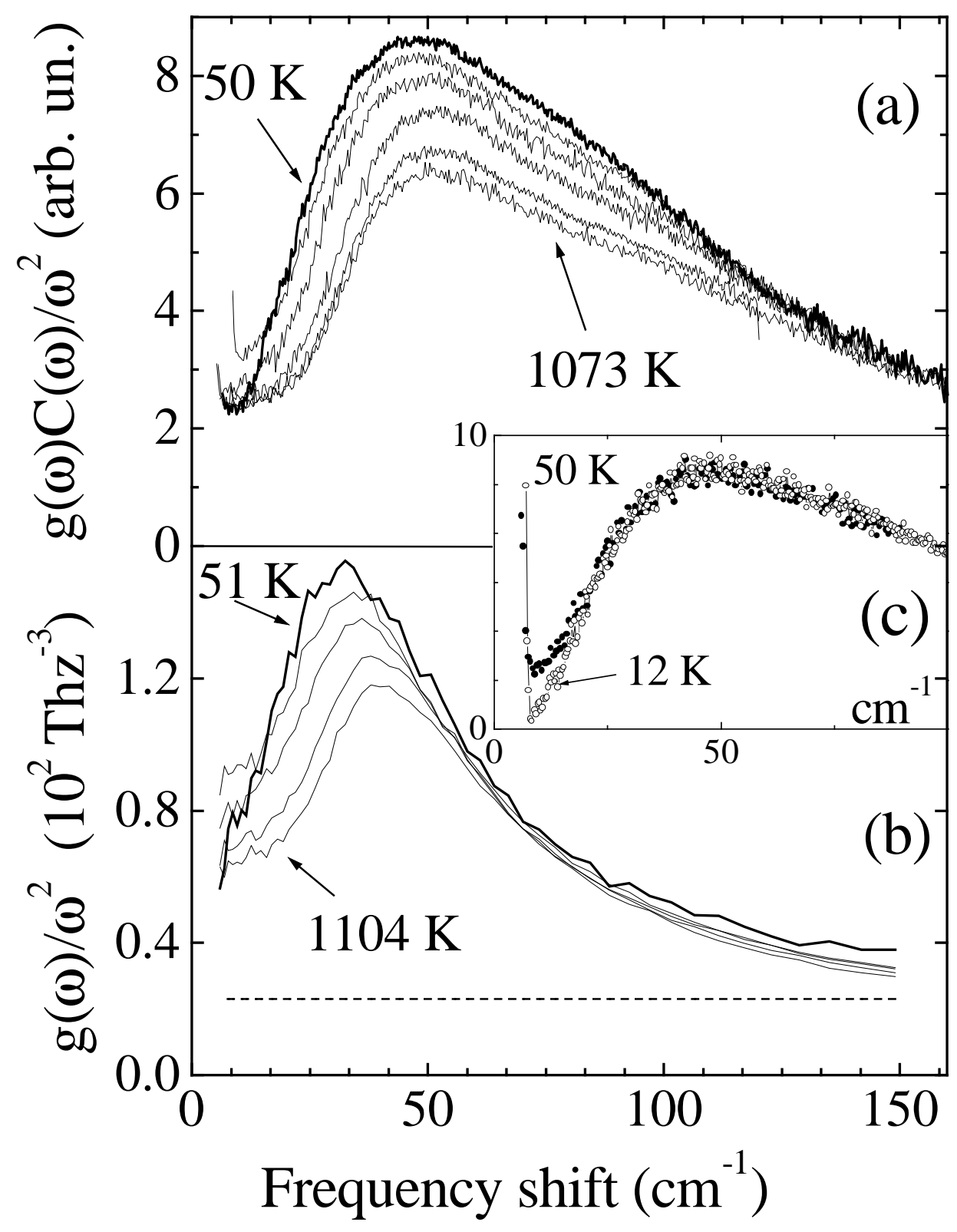

A.Fontana et al, Fig. 1 


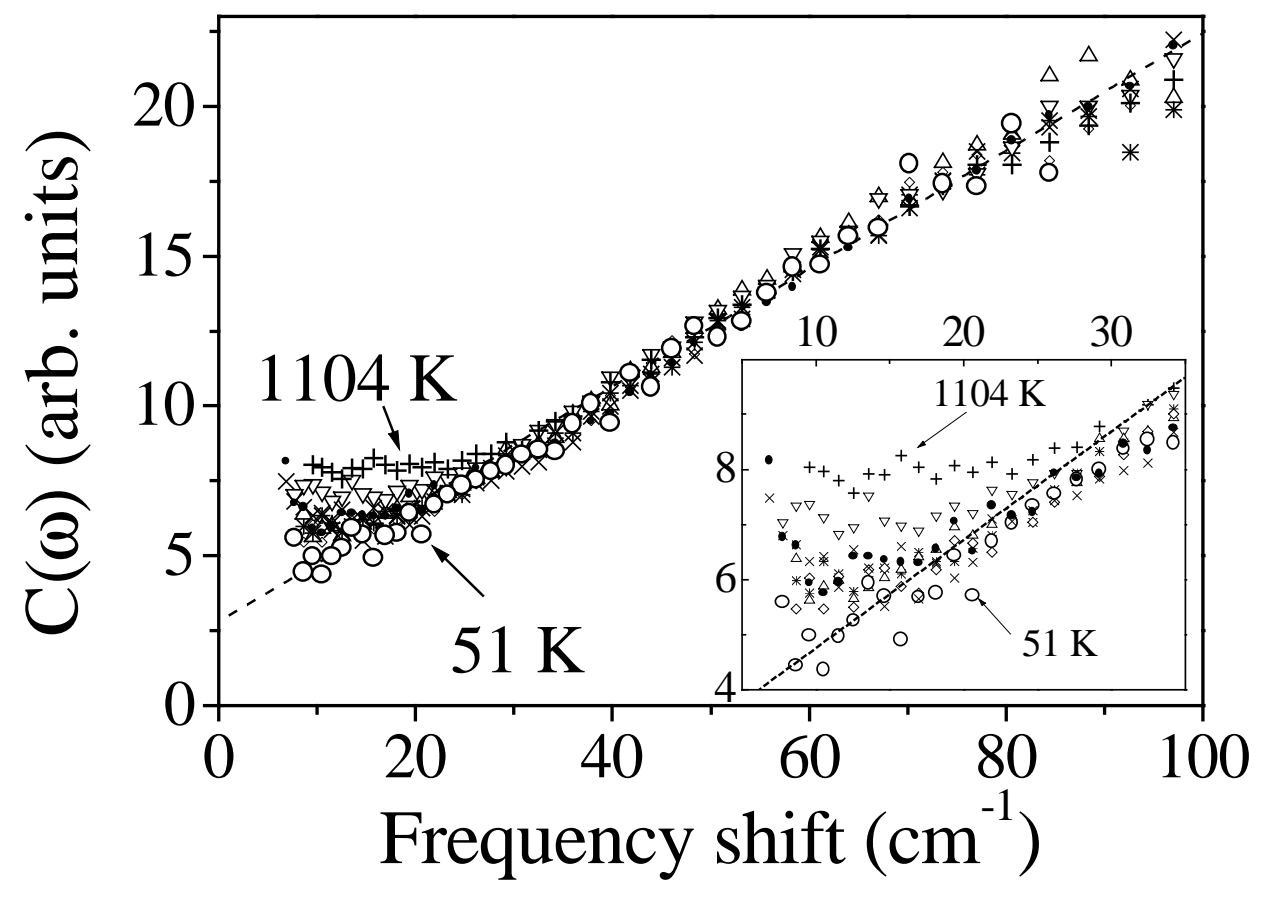

A.Fontana et al, Fig. 2 


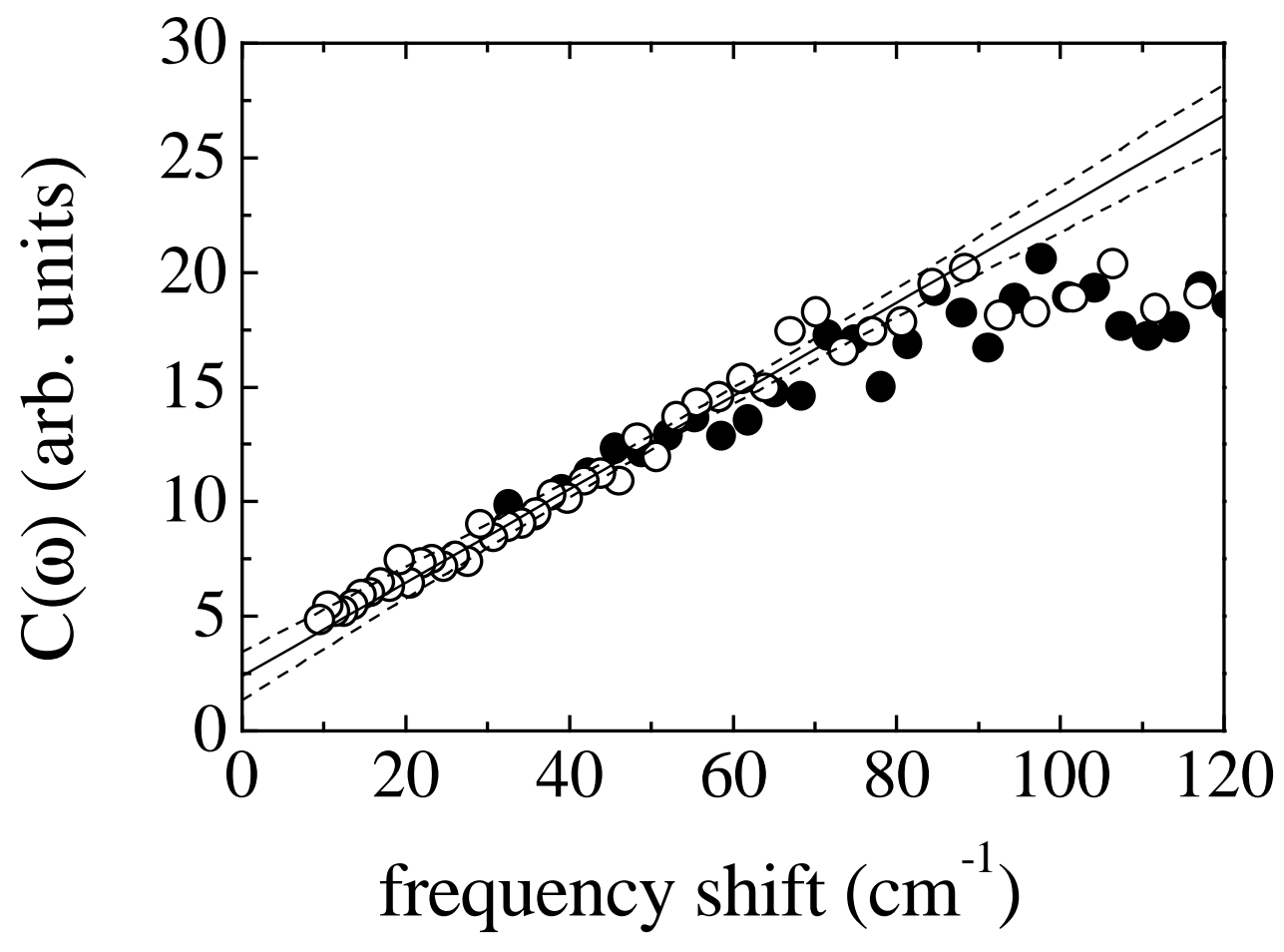

A.Fontana et al, Fig. 3 


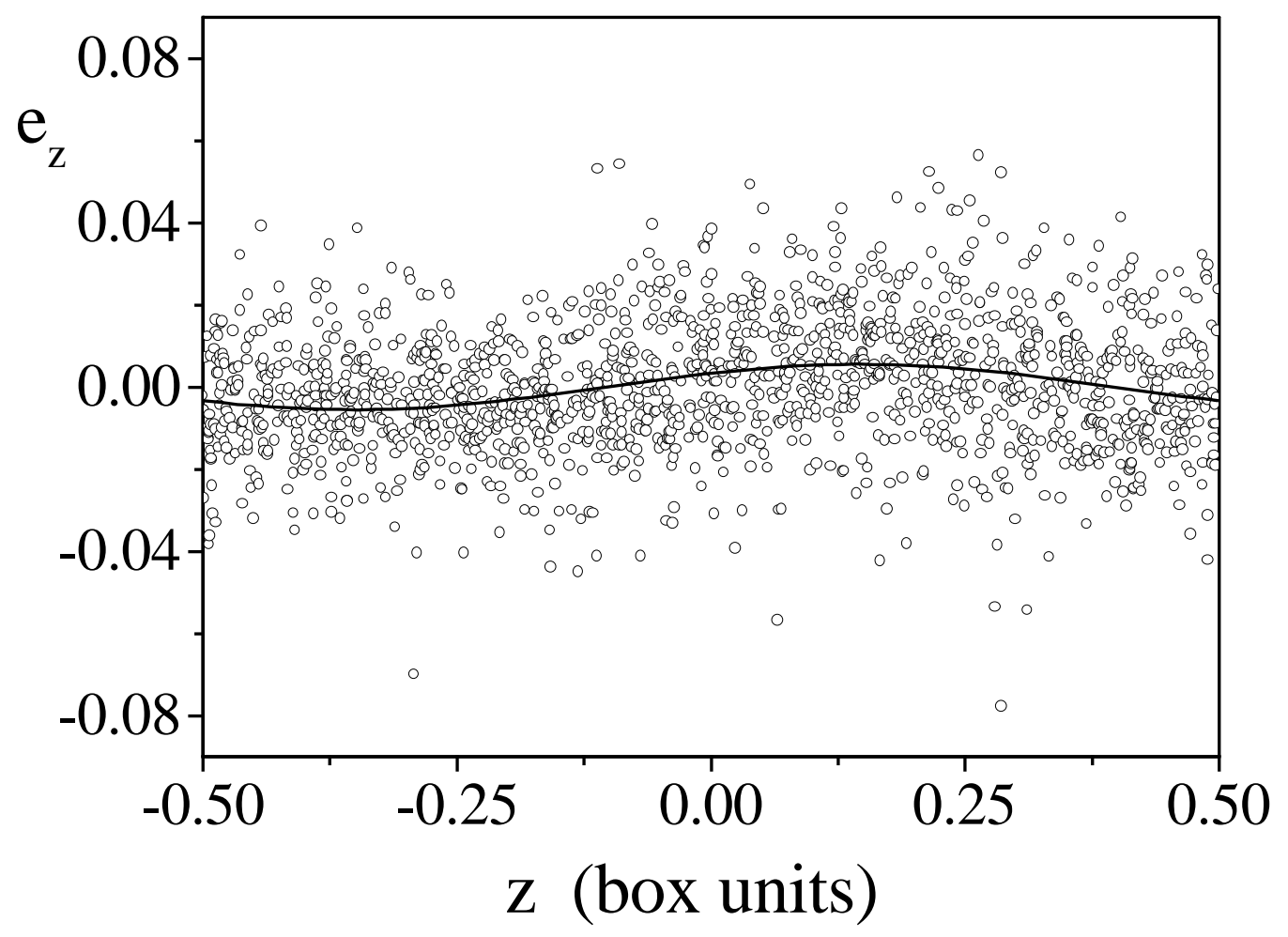

A.Fontana et al, Fig. 4 